\title{
Histopathological Analysis and Correlation of Ki67 and Progesterone Receptor Status with WHO Grading In Meningiomas
}

\author{
Tamilselvi Veeramani ${ }^{1 *}$ and J. Maheswari ${ }^{2}$ \\ 'Department of Pathology, Karpagam Faculty of Medical Sciences and Research, Othakkalmandabam, Coimbatore. India \\ ${ }^{2}$ Department of Pathology, KAP DR. Vishwanathan Government Trichy Medical College, Trichy. India
}

\begin{abstract}
Background: Meningiomas are slow growing tumors that are among the most common of CNS neoplasms and form the most common CNS tumor to be reported above 35 years of age.

Methods: This retrospective study was carried out in the Department of Pathology during the period of January2011 to May2013. A total of 50 cases were graded according to the WHO 2016 grading criteria. The biopsy specimens were fixed in 10\% neutral buffered formalin, sections was stained with Hematoxylin \& Eosin. Immunohistochemistry was done with Progesterone receptor and Ki67 antibodies for selected cases.

Results: The incidence of meningiomas was $33.11 \%$ with a female sex predilection and most common in the 5 th decade. Transitional meningioma was the most common variant to occur. The incidence of WHO Grade I, Grade II and Grade III meningiomas were $88 \%$, $4 \%$ and $8 \%$ respectively. Comparison of Ki67 LI and PR score in various grades of meningiomas were done. The average Ki67 LI and PR score were $1.1 \%, 10.25 ; 6 \%, 3 ; 16 \%, 0$ in grade I, II and III meningiomas respectively. p value showed a statistically significant difference between different grades of meningiomas with respect to PR and Ki67 status. Spearman correlation showed a clearly significant inverse relationship between the two antibodies.
\end{abstract}

Conclusion: The use of immunohistochemical markers aids in determining the aggressive nature of the tumor, its recurrence potential and can be used as prognostic markers.

Keywords: Meningioma, Progesterone Receptor, Immunohistochemistry, Prognosis.

\section{Introduction}

Meningiomas are a diverse group of neoplasms derived from the arachnoidal cap cells lining the meninges and their extensions of dura. Although most are benign, their intracranial location leads to fatal consequences. ${ }^{[1]}$

According to the CBTRUS statistics 2012, meningiomas constitute to $35 \%$ of all CNS tumors and the $5 \mathrm{yr}$ survival rate being $70 \%$ for benign and $55 \%$ for malignant meningiomas. ${ }^{[2]}$ Based upon the WHO 2016 grading, meningiomas are classified as grade I, grade II (atypical) and grade III (anaplastic meningiomas). The various parameters taken into consideration for histological grading include: Cellularity, Mitotic index, Sheet like or small cell pattern, Macronucleoli with nuclear pleomorphism and Tumor necrosis. ${ }^{[3]}$ The Armed Forces Institute of Pathology declared that the criteria for malignancy in meningiomas are satisfied when the tumor displays either or both features of anaplasia and brain parenchyma invasion. Brain-invasive meningiomas are considered as equivalent to WHO Grade II neoplasms. ${ }^{[4]}$ In the 2016
WHO classification of CNS tumors, brain invasion as a histologic criteria can alone suffice for diagnosing WHO grade II / Atypical meningioma. ${ }^{[5]}$

About $10-15 \%$ of all meningiomas are considered to be malignant. Even though grade I meningiomas are considered as benign, recurrence is seen in about $7-20 \%$ cases despite complete resection. ${ }^{[6]}$ The incidences of recurrence for atypical and anaplastic meningiomas are $29-40 \%$ and $50-78 \%$ respectively. ${ }^{[7]}$

Thus, WHO grading based on the histopathological features alone has certain limitations in predicting the exact behavior of meningiomas. Hence, the use of immunohistochemical markers aids in determining the aggressive nature of the tumor and its recurrence potential. Ki67 \& PR are the markers used to determine the nature of meningiomas. Ki67 is a non-histone intranuclear protein expressed within the proliferating cells of the cell cycle. Its expression is regarded as a specific marker for tissue proliferation. Hormonal receptor studies are not much done in our country. Various studies conducted in the developed 
countries points towards an inverse relationship between PR \& Ki67 status in meningiomas.

Hence, this study has been undertaken to evaluate the histological patterns along with WHO grading and to assess the role of IHC markers in determining the biological behavior of meningiomas.

\section{Materials and Methods}

This prospective study was carried out in the Department of Pathology, Thanjavur medical college during the period from January 2011 to May 2013. Ethical committee clearance was obtained from the Institutional Ethical Committee Board.

Sample size-50

Inclusion Criteria: Specimens sent with a clinical suspicion of meningiomas. All biopsy specimens sent with the clinical diagnosis of CNS tumors.

Exclusion Criteria: CNS infections, Reactive lesions and Non-neoplastic cystic lesions of CNS

Grading of tumors was done according to the WHO 2016 grading criteria. ${ }^{[5]}$.The specimens were mostly biopsies. All specimens were fixed in 10\% neutral buffered formalin followed by which routine tissue processing was done and were subjected to histopathological examination. Sections of 4-5 micron thickness were made and staining was done with Hematoxylin and Eosin. IHC with vimentin was done for 2 cases namely, rhabdoid and papillary variants to confirm their meningothelial nature. IHC with GFAP was done in a single case of Brain invasive meningioma.

Immunohistochemistry was done using antibodies against Ki67 and PR for about 15 randomly selected cases of various grades of meningiomas. IHC was done based on the peroxidase method using standard horse radish peroxidase kit.

Ki67 is considered a more specific marker for estimating the growth fraction and hence is the most widely used marker for determining the proliferation rate of neoplasms. ${ }^{[8]}$ Determination of Ki67 LI was done by calculating the percentage of nuclei that stains positively from regions that show maximal intensity of nuclear staining among 1000 tumor cells at high power magnification. ${ }^{[9]} \mathrm{PR}$ status is determined by a semi quantitative scoring scale based on 2 parameters namely. ${ }^{[10]}$

\section{The percentage of positive tumor cells}

0 - Absence of positively stained nuclei

$1-<10 \%$ positively stained cells in the entire section

2- $10-50 \%$ positivity
3- 51-80\% tumor nuclei show positivity

$4->80 \%$ positive tumor nuclei

Intensity of staining

0 -absent

1-weak

2-moderate

3-strong

Immunoreactive score (IRS $=0$ to 15 ) was calculated by multiplying the staining intensity and the percentage of positively stained tumor cells. ${ }^{[10]}$

Statistical analysis was performed using the SPSS software. Multivariate analysis was done using ANOVA and Kruskal-Wallis test to determine the significance of difference between various meningioma grades for both the markers Ki67 and PR. P value $<0.05$ was considered as statistically significant. Spearman correlation was done to determine the relationship between Ki67 and PR status in different grades of meningiomas.

\section{Results}

The incidence of meningiomas in our study was $33.11 \%$ with female to male ratio being $4: 1$. The most common age group affected is 50-60yrs. The most common variant (Tab.1) reported in our study is the transitional subtype accounting for $48 \%$ and the second most common variant being meningothelial meningioma with an incidence of $20 \%$ (10 cases). A single case each of papillary, Rhabdoid, anaplastic and brain invasive meningiomas (Fig.1,2) were reported. Grade I meningiomas were the predominant subtype to occur with an overall incidence of $88 \%$ (44 cases). Grade II meningiomas constitutes to $4 \%$ (2cases) and Grade III meningiomas constitutes 4 of the total 50 cases with an incidence rate of $8 \%$.A case of rhabdoid meningioma ( WHO Grade III ) was reported in a 10 year old female child. IHC with vimentin showed intense cytoplasmic positivity and confirmed the diagnosis.(Fig.3)

Table 2 shows different grades of meningioma variants and their MIB-1 LI. 15 random cases were selected and immunohistochemistry was performed on paraffinembedded sections using standard HRP kit. The study reveals rhabdoid meningioma with the highest Ki 67 score of $21 \%$ (Fig. 4 ) and the least score was observed with transitional meningioma. (0.8\%) (Fig.5)

Differences in the mean Ki67 labelling index was calculated using Anova test and the results were found to be statistically significant between various grade I, II and grade III meningiomas $(\mathrm{P}<0.001)$. 
Table 3 displays PR scoring in selected cases of meningiomas. Highest PR score was observed with the transitional and meningothelial variants.(fig 6) Least PR score of 0 was observed with the Papillary(Fig.7) and Rhabdoid (Fig.8) variants. Kruskal-Wallis test was performed to determine the difference in average PR score of various grades of meningiomas, which revealed a $P$ value of $<0.04$ and hence found to be statistically significant.

TABLE 1: Incidence of Various Types of Meningiomas

\begin{tabular}{|c|l|c|c|}
\hline S.NO & HPE Diagnosis & No of cases & Percentage ( \%) \\
\hline 1 & Angiomatous meningioma & 6 & $12 \%$ \\
\hline 2 & Anapastic meningioma & 2 & $4 \%$ \\
\hline 3 & Atypical meningioma & 1 & $2 \%$ \\
\hline 4 & Brain invasive meningiomas & 1 & $2 \%$ \\
\hline 5 & Fibroblastic meningioma & 1 & $2 \%$ \\
\hline 6 & Meningothelial meningioma & 10 & $2 \%$ \\
\hline 7 & Papillary meningioma & 1 & $6 \%$ \\
\hline 8 & Psammomatous meningioma & 3 & $2 \%$ \\
\hline 9 & Rhabdoidmeningiomas & 1 & $\mathbf{2}$ \\
\hline 10 & Transitional meningioma & 24 & $\mathbf{1 0 0 \%}$ \\
\hline
\end{tabular}

Table 2: Ki 67 Labelling Index in Various Grades of Meningioma Variants

\begin{tabular}{|c|c|c|c|c|}
\hline S.NO & PATHO NO & HPE DIAGNOSIS & WHO GRADE & MIB INDEX \\
\hline 1 & $151 / 13$ & Transitional meningioma & I & $1.2 \%$ \\
\hline 2 & $318 / 13$ & Fibroblastic meningioma & 1 & $1.0 \%$ \\
\hline 3 & $1907 / 13$ & Psammomatous meningioma & 1 & $1.7 \%$ \\
\hline 4 & $1261 / 12$ & RhabdoidMeningiomas & III & $21 \%$ \\
\hline 5 & $2334 / 13$ & Psammomatous meningioma & 1 & $0.8 \%$ \\
\hline 6 & $3046 / 13$ & Angiomatous meningioma & $\mathrm{I}$ & $2.2 \%$ \\
\hline 7 & $3060 / 12$ & Meningothelial meningioma & I & $1.3 \%$ \\
\hline 8 & $3288 / 13$ & Meningothelial meningioma & I & $1.1 \%$ \\
\hline 9 & $3286 / 13$ & Transitional meningioma & I & $0.2 \%$ \\
\hline 10 & $3455 / 13$ & Transitional meningioma & 1 & $1.4 \%$ \\
\hline 11 & $264 / 12$ & Papillary meningioma & III & $11 \%$ \\
\hline 12 & $3209 / 13$ & Brain invasive meningiomas & II & $6 \%$ \\
\hline 13 & $3553 / 13$ & Psammomatous meningioma & $\mathrm{I}$ & $1.1 \%$ \\
\hline 14 & $4113 / 13$ & Angiomatous meningioma & 1 & $0.9 \%$ \\
\hline 15 & $1866 / 11$ & Transitional meningioma & 1 & $0.4 \%$ \\
\hline
\end{tabular}

Table 3: Pr Scoring in Different Grades of Meningioma Variants.

\begin{tabular}{|c|c|c|c|c|c|c|}
\hline \multirow[t]{2}{*}{ S.NO } & \multirow{2}{*}{$\begin{array}{c}\text { PATH } \\
\text { NO }\end{array}$} & \multirow[t]{2}{*}{ HPE DIAGNOSIS } & \multirow{2}{*}{$\begin{array}{c}\text { WHO } \\
\text { GRADE }\end{array}$} & \multicolumn{3}{|c|}{ PR (0-15) } \\
\hline & & & & Intensity Score (IS) & Proportion score (PS) & IRS $=$ IS $\times$ PS \\
\hline 1 & $151 / 13$ & Transitional Meningioma & I & 3 & 3 & 9 \\
\hline 2 & $318 / 13$ & Fibroblastic Meningioma & I & 2 & 5 & 10 \\
\hline 3 & $1907 / 13$ & Psammomatous Meningioma & $\mathrm{I}$ & 2 & 4 & 8 \\
\hline 4 & $1261 / 12$ & RhabdoidMeningiomas & III & 0 & 0 & 0 \\
\hline 5 & $2334 / 13$ & Psammomatous Meningioma & I & 3 & 4 & 12 \\
\hline 6 & $3046 / 13$ & Angiomatous Meningioma & I & 2 & 4 & 8 \\
\hline 7 & $3060 / 13$ & Meningothelial Meningioma & I & 3 & 4 & 12 \\
\hline 8 & $3288 / 13$ & Meningothelial Meningioma & I & 2 & 5 & 10 \\
\hline 9 & $3286 / 13$ & Transitional Meningioma & $\mathrm{I}$ & 3 & 5 & 15 \\
\hline 10 & $3455 / 13$ & Transitional Meningioma & 1 & 2 & 4 & 8 \\
\hline
\end{tabular}




\begin{tabular}{|c|c|c|c|c|c|c|}
\hline S.NO & $\begin{array}{c}\text { PATH } \\
\text { NO }\end{array}$ & HPE DIAGNOSIS & $\begin{array}{c}\text { WHO } \\
\text { GRADE }\end{array}$ & \multicolumn{3}{|c|}{ PR (0-15) } \\
\hline 11 & $264 / 12$ & Papillary Meningioma & III & Intensity Score (IS) & Proportion score (PS) & IRS= IS x PS \\
\hline 12 & $3209 / 13$ & Brain InvasiveMeningioma & II & 1 & 0 & 0 \\
\hline 13 & $3553 / 13$ & Psammomatous Meningioma & I & 3 & 3 & 3 \\
\hline 14 & $4113 / 13$ & Angiomatous Meningioma & I & 2 & 3 & 9 \\
\hline 15 & $1866 / 11$ & Transitional Meningioma & I & 3 & 5 & 10 \\
\hline
\end{tabular}

Table 4: Comparison of Ki 67 Li And Pr Score in Various Grades of Meningiomas.

\begin{tabular}{|c|c|c|c|c|}
\hline S.NO & WHO GRADE & Average Ki67 LI & Average PR score & NO OF CASES \\
\hline 1 & $\mathrm{I}$ & $1.1 \%$ & $10.25 \%$ & 12 \\
\hline 2 & $\mathrm{II}$ & $6 \%$ & $3 \%$ & 1 \\
\hline 3 & $\mathrm{III}$ & $16 \%$ & $0 \%$ & 2 \\
\hline
\end{tabular}

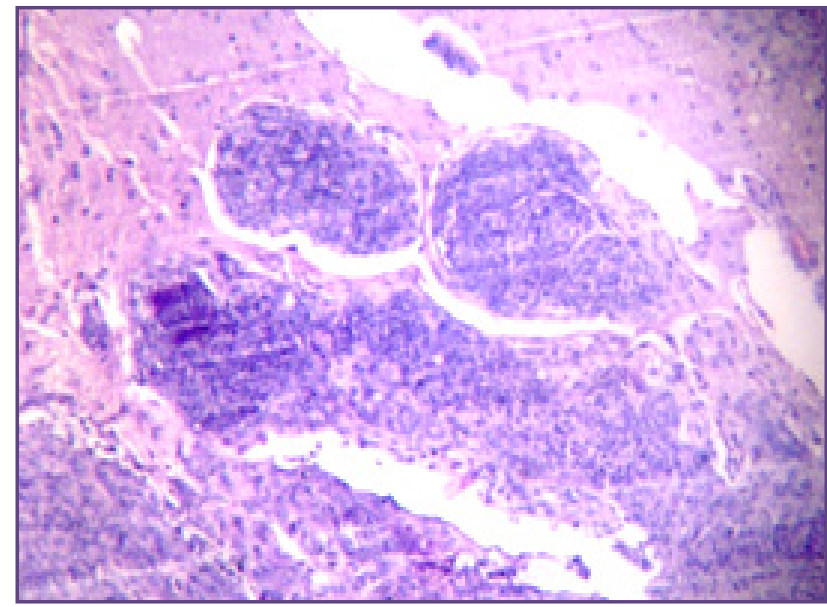

Fig. 1: Brain invasive meningioma showing irregular protrusion of tumor cells infiltrating the brain.

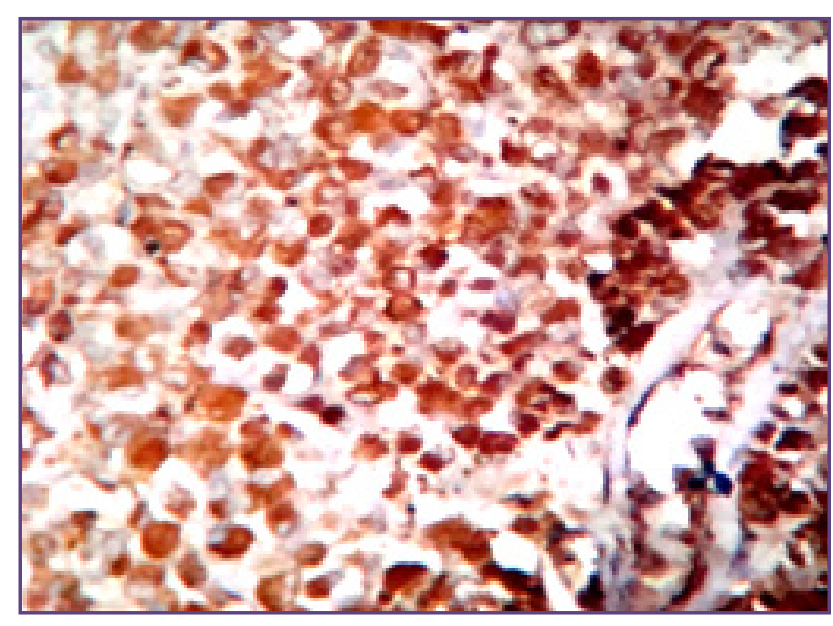

Fig. 3: Rhabdoid meningioma showing strong cytoplasmic immunoreactivity for vimentin, (40X).

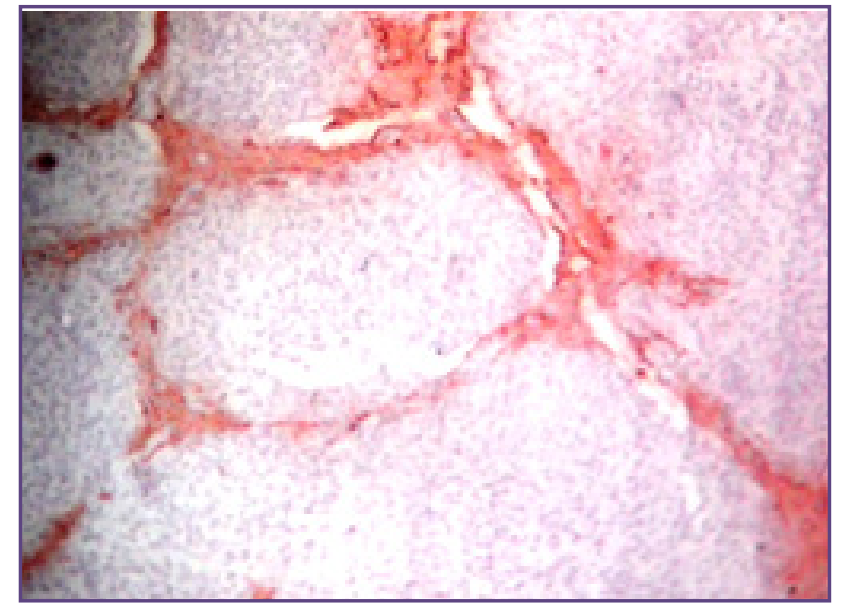

Fig. 2: Brain invasive meningioma- GFAP highlights entrapped fragments of brain parenchyma between the tumor cells, H \& E, (10X)

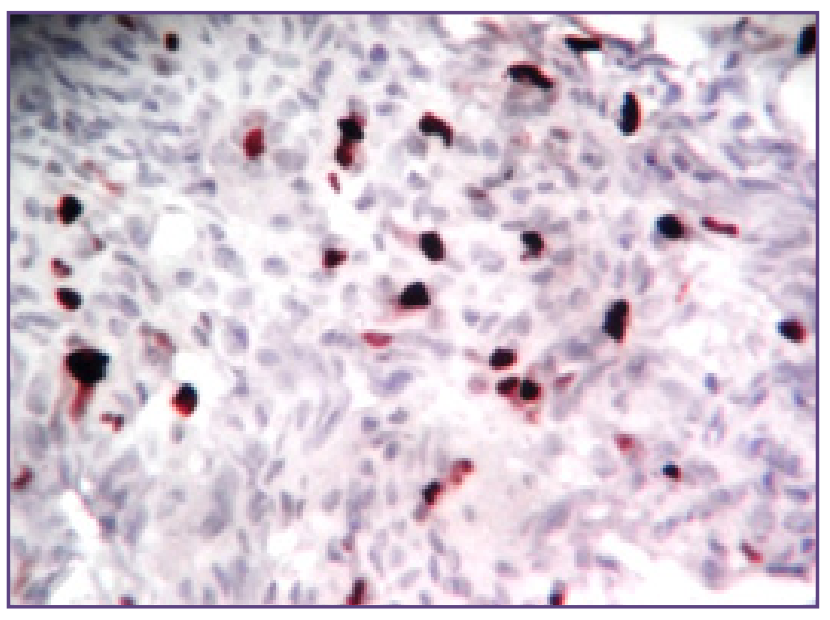

Fig. 4:Rhabdoid meningioma - Grade III, Ki67 LI of 21\% (40X). 


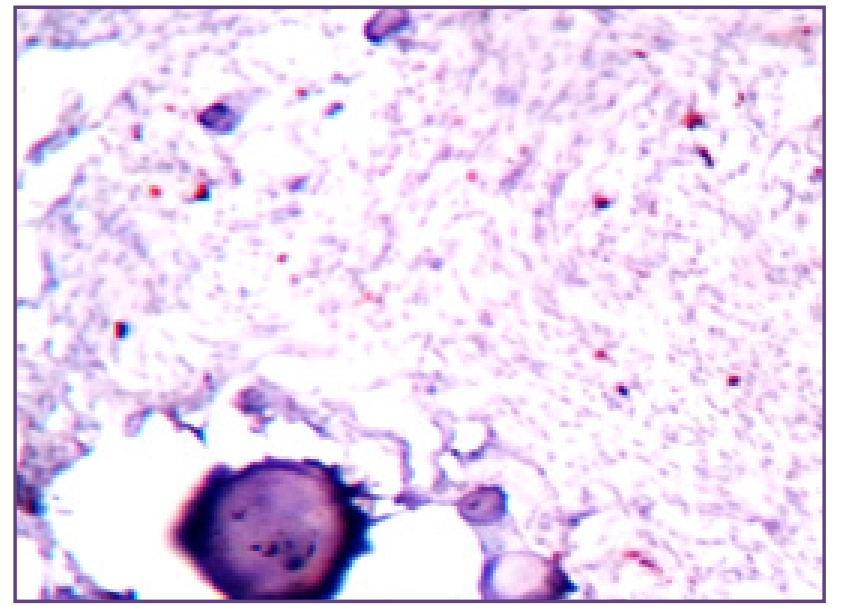

Fig. 5: Transitional meningioma - Grade I with Ki67 LI of $<0.1 \%$ (10X).

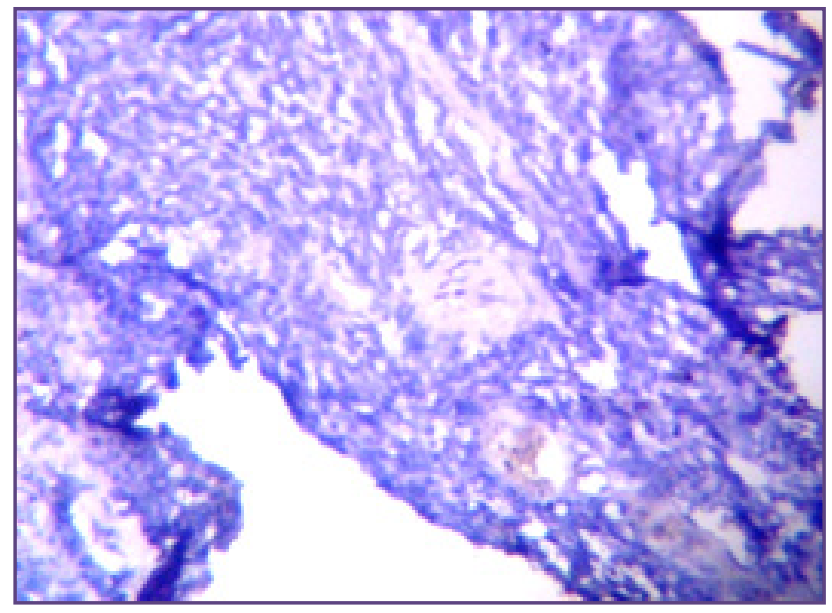

Fig. 7: Papillary meningioma - Grade III with PR score of 0 (10X).

\section{Discusson}

Meningiomas are the most common non - glial primary brain tumor. Our study showed a clear female predominance with an incidence of $80 \%$ which is similar to various other studies (CBTRUS statistical report 2012). ${ }^{[2]}$ Transitional meningiomas are the most common subtype with an incidence of $48 \%$ (Table 1) whereas the study by Sanghamithra et al [11] showed meningothelialmeningiomas as the most frequent type of occurrence. According to Sameh Ahmed et al ${ }^{[12]}$ and SashidharBabu et al ${ }^{[13]}$, grade III meningiomas showed the least rate of occurrence. In contrast our study showed grade II to be the least common type (4\%).

Our study points out that Grade I meningiomas are the commonest to occur with a female sex predilection. This was found to be similar with majority of the studies (Sanghamithra et $\mathrm{al}^{[11]}$, Intisar S.H.Patty et $\mathrm{al}^{[14]}$,

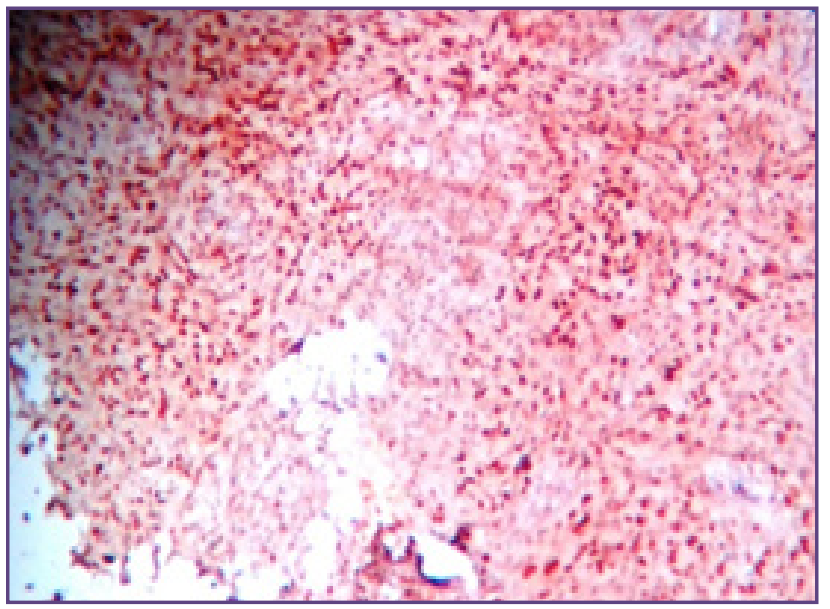

Fig. 6: Transitional meningioma- Grade I, PR score of 12 (10X).

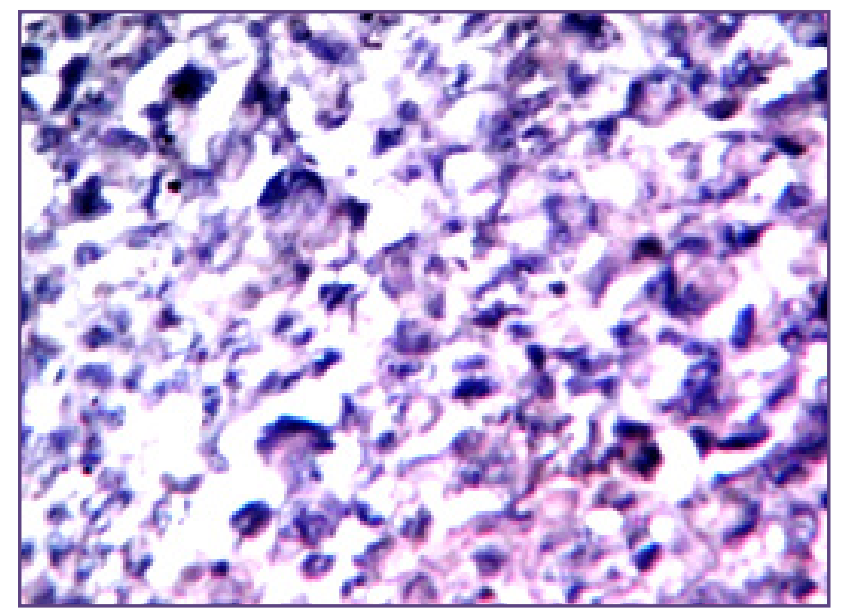

Fig. 8:Rhabdoid meningioma - Grade III showing PR score of $0(40 X)$.

Konstantinos Violaris et $\mathrm{al}^{[15]}$ ). The study on receptors of sex hormones is of great interest, given the female preponderance in meningiomas. Studies suggest that the expression of $\mathrm{PR}$ is roughly inversely proportional to the WHO histological grade. In addition to this, PR scoring also helps in assessing those cases under grade I meningiomas that are likely to recur. ${ }^{[15]}$

Ki 67 -Grading based upon the histopathological features pose certain limitations in determining the exact biological behavior of meningiomas. Mitotic count alone cannot provide adequate details regarding the aggressive nature of a tumor. Identification of mitotic figures in H\&E sections is hampered by various factors. Hence, use of Ki67 acts as an independent prognostic factor in predicting the biological behavior. Ki 67 is an intranuclear protein that is expressed within proliferative phases of the cell cycle. The monoclonal 
antibody against Ki 67, namely the MIB-I labelling index is used to predict the recurrence potential and aggressive nature of meningiomas. Generally, higher the Ki 67 index, higher is the grade of meningiomas and its tendency for recurrence ${ }^{[16]}$. Hence, PR when used in conjunction with Ki67 LI may act as useful ancillary markers in determining the prognosis and grading of meningiomas.

Our study showed almost similar Ki67 labelling indices as in the studies of Sanghamithra et $\mathrm{al}^{[11]}$ and Intisar Patty et al ${ }^{[14]}$. The Ki67 LI correlated well with the increasing grades of meningiomas and this is in accordance with the studies conducted by Amatya et a ${ }^{[16]}$ and Nasrin Shyanfaret et al. ${ }^{\left[{ }^{[7]}\right.}$ They showed that Grade I meningiomas are the one with highest PR expression seen in about $97 \%$, Grade II and grade III meningiomas with $20 \%$ and $0 \%$ positivity respectively. Similar results were obtained with our present study too. Brandis et al ${ }^{[18]}$ and Wolfsberger $\mathrm{S}$ et $\mathrm{a}^{[19]}$ reported that malignant meningiomas are devoid of PR. This is in accordance with our study where rhabdoid and papillary meningiomas was PR negative with a score of 0 . Meningiomas with higher proliferative index and negative PR are more likely to be of higher grade and hence carry an increased potential forrecurrence even after complete resection.

Despite the limitations caused by the small study group chosen, Ki67 LI along with PR status in combination with WHO histopathological grading might help in identifying patients at a high risk of relapse and this early detection makes institution of more effective treatment as early as possible.

\section{Conclusion}

Progesterone receptor and Ki67 antibodies when used in addition to histopathological grading can aid in identifying the cases that are likely to recur in Grade I meningiomas. Study trials when conducted similar to our present one might help in evaluating the exact tumor burden in the country and would encompass the role of ancillary methods like immunohistochemistry in the prognostication of tumors.

\section{Acknowledgment}

We extend our gratitude to our institution departmental head for providing expertise and assistance. We thank all the references cited. We are grateful to our family who supported us in our work.

\section{References}

1. Claus EB, Bondy ML, Schildkraut JM, Wiemels JL, Wrensch M, Black PM. Epidemiology of intracranial meningioma. Neurosurgery. 2005 Dec;57(6):1088-1095; discussion 1088-1095.

2. Ostrom QT, Gittleman H, Fulop J, Liu M, Blanda R, Kromer C, et al. CBTRUS Statistical Report: Primary Brain and Central Nervous System Tumors Diagnosed in the United States in 2008-2012. Neuro-oncology. 2015 Oct;17Suppl 4:iv1-iv62.

3. Commins DL, Atkinson RD, Burnett ME. Review of meningioma histopathology. Neurosurg Focus. 2007;23(4):E3.

4. Perry A, Brat DJ. Philadelphia: Churchill Livingstone; 2010. Practical Surgical Neuropathology: A diagnostic approach; pp. 185-218.

5. Louis DN, Perry A, Reifenberger G, von Deimling A, Figarella-Branger D, Cavenee WK, et al. The 2016 World Health Organization Classification of Tumors of the Central Nervous System: a summary. ActaNeuropathol. 2016 Jun;131(6):803-20.

6. Choi SJ, Chang ED, Kwon SO, Kye DK, Park CK, Lee SW, et al. Comparison of Proliferative Activity in Each Histological Subtypes of Benign and Atypical Intracranial Meningiomas by PCNA and Ki-67 Immunolabeling. Journal of Korean Neurosurgical Society. 2000 Sep 1;29(9):1215-21.

7. Yang S-Y, Park C-K, Park S-H, Kim DG, Chung YS, Jung H-W. Atypical and anaplastic meningiomas: prognostic implications of clinicopathological features. J NeurolNeurosurgPsychiatr. 2008 May;79(5):574-80.

8. Shrestha P, Shrestha I, Kurisu K. Usefulness of Ki-67 in the histological evaluation of neoplastic lesions of central nervous system. Journal of Institute of Medicine. 2008;30(1):68-71.

9. Karabağli P, Sav A. Proliferative indices (MIB-1) in meningiomas: Correlation with the histological subtypes and grades. Journal of Neurological Sciences [Turkish]. 2006; 23(4):279-86.

10. Roser F. The prognostic value of progesterone receptor status in meningiomas. Journal of Clinical Pathology. 2004 Oct 1;57(10):1033-7.

11. Chatterjee U, Mukherjee S, Ghosh S, Chatterjee S. Detection of progesterone receptor and the correlation with Ki-67 labeling index in meningiomas. Neurology India. 2011;59(6):817.

12. Sakr SA, Salem M. Atypical meningioma: Clinicopathological analysis of a new WHO classification. Pan Arab Journal of Neurosurgery. 2011;15(1):36-41.

13. Challa S, Babu S, Uppin S, Uppin M, Panigrahi M, Saradhi $\mathrm{V}$, et al. Meningiomas: Correlation of Ki67 with histological grade. Neurology India. 2011;59(2):204.

14. Intisar s.h. Patty. Central nervous system tumors a clinicopathological study Kurdistan 1st conference on biological sciences j. dohuk Univ., 2008;11, no. 1,. 
15. Violaris K, Katsarides V, Sakellariou P. The Recurrence Rate in Meningiomas: Analysis of Tumor Location, Histological Grading, and Extent of Resection. Open Journal of Modern Neurosurgery. 2012;02(01):6-10.

16. Amatya VJ, Takeshima Y, Sugiyama K, Kurisu K, Nishisaka T, Fukuhara T, et al. Immunohistochemical study of Ki-67 (MIB-1), p53 protein, p21WAF1, and p27KIP1 expression in benign, atypical, and anaplastic meningiomas. Hum Pathol. 2001 Sep;32(9):970-5.

17. Shayanfar N, Mashayekh M, Mohammadpour M. Expression of Progestrone Receptor and Proliferative Marker ki 67, in Various Grades of Meningioma. ActaMedicaIranica. 2010;48(3):142-7.

18. Brandis A, Mirzai S, Tatagiba M, Walter GF, Samii M, Ostertag H. Immunohistochemical detection of female sex hormone receptors in meningiomas: correlation with clinical and histological features. Neurosurgery. 1993 Aug;33(2):212-217; discussion 217-218.

19. Wolfsberger S, Doostkam S, Boecher-Schwarz H-G, Roessler K, van Trotsenburg M, Hainfellner JA, et al. Progesterone-receptor index in meningiomas: correlation with clinico-pathological parameters and review of the literature. Neurosurg Rev. 2004 Oct;27(4):238-45.

*Corresponding author:

DR. V. Tamilselvi, Assistant Professor, Department of Pathology, Karpagam Faculty of Medical Sciences and Research, Othakkalmandabam, Coimbatore.-641032, Tamilnadu. INDIA, PINCODE-641032.

Phone: +91 8903489000

Email: tamilselviaruna16@gmail.com

Date of Submission : 26.04.2017

Financial or other Competing Interests: None. 